\title{
Multicurves and regular functions on the representation variety of a surface in $\mathrm{SU}(2)$
}

Laurent Charles and Julien Marché

\begin{abstract}
Given a compact surface $\Sigma$, we consider the representation space

$$
\mathcal{M}(\Sigma)=\operatorname{Hom}\left(\pi_{1}(\Sigma), \operatorname{SU}(2)\right) / \operatorname{SU}(2) .
$$

We show that the trace functions associated to multicurves on $\Sigma$ are linearly independent as functions on $\mathcal{M}(\Sigma)$. The proof relies on the Fourier decomposition of the trace functions with respect to a torus action on $\mathcal{M}(\Sigma)$ associated to a pants decomposition of $\Sigma$. Consequently the space of trace functions is isomorphic to the Kauffman skein algebra at $A=-1$ of the thickened surface.
\end{abstract}

Mathematics Subject Classification (2010). 57M27, 57M25, 37E30, 81S10.

Keywords. Representation variety, multicurve, skein algebra, Dehn coordinates, topological quantum field theory.

\section{Introduction}

Given a compact and oriented surface $\Sigma$, one defines its representation space as the quotient $\operatorname{Hom}\left(\pi_{1}(\Sigma), G\right) / G$. For $G=\operatorname{Sl}(2, \mathbb{C}), \operatorname{SU}(2), \operatorname{Sl}(2, \mathbb{R})$, we obtain three related and celebrated spaces. The first one is an algebraic variety which classifies semi-stable complex bundles of rank 2 over $\Sigma$ with trivial determinant: it contains the two other ones. The second space is compact and contains an open and dense subset supporting a natural symplectic form. Its quantization provides a construction of a topological quantum field theory (TQFT) which has interesting interactions with the topology of 3-manifolds. Finally the last space contains as a connected component the Teichmüller space, that is, the moduli space of hyperbolic structures on $\Sigma$.

The purpose of this article is to study a special class of functions on these spaces called "trace functions". Given a 1-dimensional submanifold $\gamma$ of $\Sigma$ and a representation $\rho \in \operatorname{Hom}\left(\pi_{1}(\Sigma), G\right) / G$ one sets

$$
f_{\gamma, G}([\rho])=\prod_{i}\left(-\operatorname{tr}\left(\rho\left(t_{i}\right)\right)\right)
$$


where the $t_{i}$ 's represent the free homotopy classes of the components of $\gamma$.

Assume that the Euler characteristic of $\Sigma$ is negative. We prove that the functions $f_{\gamma, G}$ where $\gamma$ runs over the isotopy classes of 1-submanifolds of $\Sigma$ without a component bounding a disc are linearly independent as functions on $\operatorname{Hom}\left(\pi_{1}(\Sigma), G\right) / G$ for $G=\mathrm{SU}(2)$ or $\mathrm{Sl}(2, \mathbb{C})$. In the case $G=\mathrm{Sl}(2, \mathbb{C})$, it follows that these functions form a basis of the coordinate ring of the representation variety. Consequently the coordinate ring is isomorphic to the Kauffman bracket skein algebra of $\Sigma \times[0,1]$ at $A=-1$, cf. Sections 1.1 and 1.2 for precise statements.

D. Bullock showed in [Bul] that the last assertion is equivalent to proving that the Kauffman bracket skein algebra has no non-zero nilpotents. Bullock's proof relies on a delicate analysis of algebraic relations between trace functions which started in [GM]. This statement also follows from Theorem 2.8 and 7.1 of [PS]. Our strategy is completely different and somewhat simpler: using a pants decomposition of the surface, we define on $\mathcal{M}(\Sigma)=\operatorname{Hom}\left(\pi_{1}(\Sigma), \mathrm{SU}(2)\right) / \mathrm{SU}(2)$ an action of the torus $\mathbb{T}^{\mathscr{C}}$ where $\mathscr{C}$ is the set of separating curves of the decomposition. Moreover, 1-dimensional submanifolds of $\Sigma$ are parametrized up to isotopy by their Dehn coordinates, which form a system of parameters depending on the pants decomposition. Finally, we compute the Fourier decomposition of the trace functions relatively to the action of $\mathbb{T}^{\mathscr{C}}$ and show that one can recover the geometric intersection number of two curves and more generally the full Dehn parameters of a multicurve via its Fourier decomposition. This allows us to prove our assertion.

Our motivation to study the trace functions is the quantization of the representation space $\mathcal{M}(\Sigma)$. First, the space of trace functions is a Poisson algebra, the Poisson bracket being defined with the symplectic structure of Atiyah-Bott [AB]. Hence the skein algebra at $A=-1$ inherits a Poisson bracket. It appears that the skein algebra at $A=e^{-i \hbar / 4}$ is a deformation quantization of this Poisson algebra. This is a consequence of the Goldman formula [Gol] expressing the bracket of trace functions, cf. $[\mathrm{BFK}]$ and $[\mathrm{T}]$.

Not only do we have a formal quantization, but also a strict quantization provided by the topological quantum field theory. Working with the combinatorial version of TQFT [BHMV] we associate to $\Sigma$ a family of Hilbert spaces $V_{k}(\Sigma)$ and to each curve $\gamma$ a family of operators $\left(\mathrm{Op}_{k}(\gamma): V_{k}(\Sigma) \rightarrow V_{k}(\Sigma)\right)$. Then it appears that the natural symbol of this family of operators is the trace function of $\gamma$. Indeed, by [MN] the asymptotic behavior of $\mathrm{Op}_{k}(\gamma)$ as the level $k$ tends to infinity is controlled at first order by the trace function. Furthermore the composition and the commutator of operators corresponds to the product and Poisson bracket of the trace functions. So the relation between the curve operators and the trace functions is similar to the one in microlocal analysis between operators and their symbols. From this point of view, our result produces non-vanishing trace functions and consequently non-vanishing curve operators. This has non-trivial consequences like the asymptotic faithfulness of the representation of the mapping class group on $V_{k}(\Sigma)$ provided by TQFT, cf. [And], [FWW], [MN]. 
In the next parts of this introduction, we give precise statement of our results (Sections 1.1 and 1.2) and of the quantization of $\mathcal{M}(\Sigma)$ (Sections 1.3 and 1.4). Note that we do not use skein modules and TQFT in the sequel of the paper. So the remainder of the paper is completely independent of Sections 1.3 and 1.4. Section 2 introduces the Dehn coordinates for multicurves on a surface while Section 3 describe torus actions on $\operatorname{Hom}\left(\pi_{1}(\Sigma, \mathrm{SU}(2)) / \mathrm{SU}(2)\right.$ which are part of the action-angle coordinates of [JW2]. In Section 4, we give the main ingredients for computing the Fourier coefficients of the trace functions. Section 5 describes the applications of the preceding computations while Section 6 explains the isomorphism of the trace functions algebra with the coordinate ring of the representation variety in $\operatorname{Sl}(2, \mathbb{C})$.

After we completed this article, A. Sikora mentioned us that a very different proof of Theorem 1.1 appears in Corollary 50 of [Si].

Acknowledgment. This work originated with an exciting workshop in La Llagone in January 2008. The second author received support ANR-08-JCJC-0114-01 from Agence Nationale de la Recherche.

1.1. Results. Let $G$ be the group $\operatorname{SU}(2)$ or $\operatorname{Sl}(2, \mathbb{C})$. Now consider the space $\operatorname{Hom}(\pi, G) / G$ of morphisms from a group $\pi$ to $G$ up to conjugation. For any $t \in \pi$, introduce the function $\chi_{t}$ of $\operatorname{Hom}(\pi, G) / G$ defined by

$$
\chi_{t}([\rho])=-\operatorname{tr}(\rho(t)), \quad \rho \in \operatorname{Hom}(\pi, G) .
$$

Let us call a trace function any linear combination of the $\chi_{t}$ 's with complex coefficients. Because of the relation

$$
\operatorname{tr}(a) \operatorname{tr}(b)=\operatorname{tr}(a b)+\operatorname{tr}\left(a^{-1} b\right) \quad \text { for all } a, b \in G
$$

the space $\mathcal{T}(\pi, G)$ of trace functions is a subalgebra of the algebra of complex valued functions of $\operatorname{Hom}(\pi, G) / G$.

When $\pi$ is of finite type, $\mathcal{T}(\pi, \operatorname{Sl}(2, \mathbb{C}))$ is finitely generated and is the coordinate ring of the $\operatorname{Si}(2, \mathbb{C})$ character variety of $\pi$. The relation with the usual definition is explained in Part 6.

Assume now that $\pi$ is the fundamental group of a manifold $M$. Let us define a trace function $f_{\gamma, G}$ for any isotopy class $\gamma$ of 1-dimensional compact submanifold of $M$ without arc components. Let $t_{1}, \ldots, t_{n}$ be the homotopy classes of loops which are freely homotopic to the connected component of a representant of $\gamma$ and set

$$
f_{\gamma, G}=\chi_{t_{1}} \ldots \chi_{t_{n}} .
$$

Since the $t_{i}$ 's are well defined up to conjugation and inversion, this product only depends on the isotopy class $\gamma$.

We will use these definitions in two cases. First, $M$ is a surface and $\gamma$ the isotopy class of a multicurve, that is no component of $\gamma$ bounds a disc. Secondly, $M$ is 3dimensional, the 1-dimensional compact submanifolds without arc components are then called the links of $M$. 
Theorem 1.1. Let $G$ be $\mathrm{SU}(2)$ or $\mathrm{Sl}(2, \mathbb{C})$ and $\pi$ the fundamental group of a compact orientable surface $\Sigma$ with boundary (possibly empty) and negative Euler characteristic. Then the trace functions $f_{\gamma, G}$, where $\gamma$ runs over the isotopy classes of multicurves of $\Sigma$ which do not meet the boundary, form a basis of $\mathcal{T}(\pi, G)$.

Some elements of the fundamental group of $\Sigma$ cannot be represented by multicurves because of the self-crossings. However, using the relations (1), one can show easily that the trace functions $\left(f_{\gamma, G}\right)$ are a spanning set of $\mathcal{T}(\pi, G)$. The proof that they are linearly independent is much more delicate and is the content of Theorem 5.3. Let $j$ be the obvious map

$$
\operatorname{Hom}(\pi, \operatorname{SU}(2)) / \operatorname{SU}(2) \rightarrow \operatorname{Hom}(\pi, \operatorname{Sl}(2, \mathbb{C})) / \operatorname{Sl}(2, \mathbb{C}) .
$$

By the previous theorem, $j^{*}$ maps $\mathcal{T}(\pi, \operatorname{Sl}(2, \mathbb{C}))$ bijectively onto $\mathcal{T}(\pi, \mathrm{SU}(2))$.

1.2. The Kauffman bracket skein module at $\boldsymbol{A}=\mathbf{- 1}$. Let $M$ be a 3-dimensional oriented compact manifold. We call banded link an orientation preserving embedding of a finite disjoint union of annuli in $M$. Note that these annuli are not framed as we do not fix an orientation of their core. The skein module of $M$ is defined as the module over $\mathbb{C}\left[A, A^{ \pm 1}\right]$ freely generated by the set of isotopy classes of banded links of $M$ quotiented by the relations of Figure 1 .

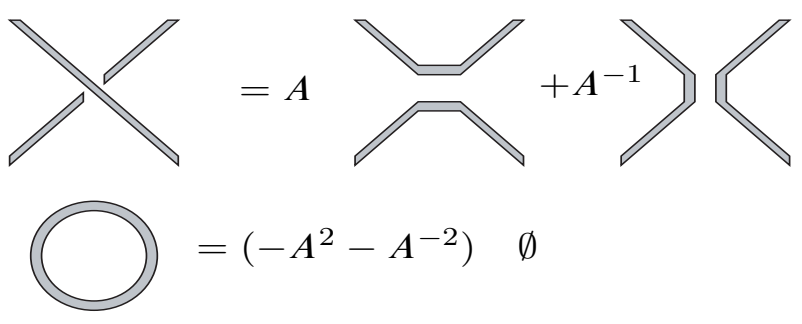

Figure 1. Kauffman bracket skein relations.

We will denote this module by $\mathcal{K}(M, A)$. If we replace $A$ by -1 we get a vector space that we denote by $\mathcal{K}(M,-1)$. As a consequence of the relation (1), we have a well-defined map

$$
\mathcal{K}(M,-1) \rightarrow \mathcal{T}(\pi, G)
$$

sending a link $\gamma$ to $f_{\gamma, G}$. Here $\pi$ is the fundamental group of $M$.

Assume that $M=\Sigma \times[0,1]$ where $\Sigma$ is an orientable compact surface. Then by identifying $\Sigma$ with $\Sigma \times\{1 / 2\} \subset M$, each multicurve of $\Sigma$ defines a link in $M$. Any link $L$ in $M$ has a generic projection to $\Sigma$ : by choosing such a projection and applying the Kauffman relation at each crossing, we get a decomposition of $L$ as a linear combination of multicurves. Two projections are related by a sequence of 
Reidemeister moves and one can show that the decomposition of $L$ does not depend on the projection by analyzing each move. Hence, the family of isotopy classes of multicurves of $\Sigma$ is a basis of $\mathcal{K}(M,-1)$. As a corollary of Theorem 1.1, we obtain

Theorem 1.2. For any compact orientable surface $\Sigma$, the natural map

$$
\mathcal{K}(\Sigma \times[0,1],-1) \rightarrow \mathcal{T}(\pi, G)
$$

is an isomorphism.

Bullock proved in [Bul] that the kernel of this map consists of the nilradical of $\mathcal{K}(\Sigma \times[0,1],-1)$. Moreover, it is claimed without proof in [PS] that this nilradical is zero.

The skein module $\mathcal{K}(\Sigma \times[0,1],-1)$ is an algebra where the product is defined in the following way: let $\iota: \Sigma \times[0,1] \amalg \Sigma \times[0,1] \rightarrow \Sigma \times[0,1]$ be the map defined by $(x, t) \mapsto\left(x, \frac{1+t}{2}\right)$ for the first copy and $(x, t) \mapsto\left(x, \frac{t}{2}\right)$ for the second one. Then we set

$$
\gamma \cdot \delta=\iota(\gamma \amalg \delta) .
$$

As a consequence of the relations (1) this product is sent to the product of functions by the isomorphism of Theorem 1.2. We will refer to this product as the stacking product: it is also defined for the Kauffman skein module $\mathcal{K}(\Sigma \times[0,1], A)$.

1.3. $\mathcal{T}(\pi, G)$ as a Poisson algebra. In this subsection and the next, $G$ is the group $\mathrm{SU}(2), \Sigma$ is a closed orientable surface and $\pi$ its fundamental group. The space $\mathcal{M}=\operatorname{Hom}(\pi, G) / G$ has a natural topology such that the trace functions are continuous. The subset $\mathcal{M}^{s}$ consisting of classes of irreducible representations is open and dense. By $[\mathrm{AB}]$ and $[\mathrm{Gol}], \mathcal{M}^{s}$ is a symplectic manifold. Furthermore Goldman in [Gol] expressed the Poisson bracket of the trace functions of two curves intersecting transversally as a trace function. Consequently, $\mathcal{T}(\pi, G)$ is a Poisson subalgebra of $e^{\infty}\left(\mathcal{M}^{s}, \mathbb{C}\right)$. The Poisson bracket appears also naturally on the topological side in the following way.

Let us introduce the Kauffman module $\mathcal{K}\left(M,-e^{i \hbar / 4}\right)$ of a 3-dimensional compact oriented manifold. It is defined as the free $\mathbb{C}[[\hbar]]$-module generated by the set of isotopy classes of banded links of $M$ quotiented by the relations of Figure 1 with $A=-e^{i \hbar / 4}$.

One has a natural $\mathbb{C}$-linear map from $\mathcal{K}\left(M,-e^{i \hbar / 4}\right)$ to $\mathcal{K}(M,-1)$ sending a linear combination $\sum c_{i}(\hbar) \gamma_{i}$ of banded links to $\sum c_{i}(0) \tilde{\gamma}_{i}$, where $\tilde{\gamma}_{i}$ is the core of $\gamma_{i}$. The kernel of this map is $\hbar \mathcal{K}\left(M,-e^{i \hbar / 4}\right)$.

Assume now that $M=\Sigma \times[0,1]$. Using the isomorphism of Theorem 1.2, we obtain an exact sequence

$$
0 \rightarrow \hbar \mathcal{K}\left(M,-e^{i \hbar / 4}\right) \rightarrow \mathcal{K}\left(M,-e^{i \hbar / 4}\right) \stackrel{\sigma}{\longrightarrow} \mathcal{T}(\pi, G) \rightarrow 0
$$


Furthermore the multiplication by $\hbar$ is a bijection

$$
\mathcal{K}\left(M,-e^{i \hbar / 4}\right) \rightarrow \hbar \mathcal{K}\left(M,-e^{i \hbar / 4}\right)
$$

The injectivity follows from the fact that $\mathcal{K}\left(M,-e^{i \hbar / 4}\right)$ is free as a $\mathbb{C}[[\hbar]]-$ module, a basis being given by the family of isotopy classes of multicurves of $\Sigma$.

Finally, $\mathcal{K}\left(M,-e^{i \hbar / 4}\right)$ has a natural stacking product $*$ and a natural involution ${ }^{-}$ defined by conjugating coefficients and sending a banded link $\gamma$ to $S(\gamma)$ where $S: \Sigma \times$ $[0,1] \rightarrow \Sigma \times[0,1]$ is defined by $S(x, t)=(x, 1-t)$. One has $\overline{f * g}=\bar{g} * \bar{f}$.

Theorem 1.3. Let $G$ be the group $\mathrm{SU}(2), \Sigma$ a closed surface, $\pi$ its fundamental group and $M=\Sigma \times[0,1]$. Then the Kauffman bracket skein module $\mathcal{K}\left(M,-e^{i \hbar / 4}\right)$ with its product $*$ and involution ${ }^{-}$is a deformation of the Poisson algebra $\mathcal{T}(\pi, G)$ in the sense that for any $f, g \in \mathcal{K}\left(M,-e^{i \hbar / 4}\right)$ one has

$$
\begin{gathered}
\sigma(f * g)=\sigma(f) \sigma(g), \quad \sigma(\bar{f})=\overline{\sigma(f)}, \\
\sigma\left(\hbar^{-1}(f * g-g * f)\right)=\frac{1}{i}\{\sigma(f), \sigma(g)\}
\end{gathered}
$$

where $\sigma$ is defined in (2) and $\hbar^{-1}$ is the inverse of the map (3).

The first equation follows from the fact that the isomorphism of Theorem 1.2 is an algebra morphism. For the second equation, observe that the classes in $\mathcal{K}\left(M,-e^{i \hbar / 4}\right)$ of the multicurves of $\Sigma$ are real and that their trace functions are real too. Last formula on the commutator follows from the Goldman formula expressing the Poisson bracket of two trace functions (see [Gol]).

The Poisson bracket depends on the symplectic form on $\mathcal{M}^{s}$ which itself depends on a choice of an invariant scalar product on the Lie algebra. We set $\langle A, B\rangle=\operatorname{tr}\left(A B^{*}\right)$ for $A, B \in \mathfrak{s u}(2)$. In [AB], it is explained how a symplectic structure on $\mathcal{M}^{s}$ comes from the interpretation of $\operatorname{Hom}(\pi, G) / G$ as gauge equivalence classes of flat $G$ connections. More precisely, the symplectic form comes as a reduction of the affine space of all $G$-connections. On the latter space, the form is given by the following formula:

$$
\omega(a, b)=\int_{\Sigma}\langle a \wedge b\rangle \quad \text { for } a, b \in \Omega^{1}(\Sigma, \mathfrak{s u}(2)) .
$$

1.4. Topological quantum field theory. In this section, we give an application of our results to topological quantum field theory (TQFT). Precisely, the proof of asymptotic faithfulness of quantum representations of mapping class groups given in [MN] uses Theorem 1.2 as a final step.

Let $M=\Sigma \times[0,1]$. By sending $\sum P_{i}(A) \gamma_{i}$ to $\sum P_{i}\left(-e^{i \hbar / 4}\right) \gamma_{i}$, one identifies the skein module $\mathcal{K}(M, A)$ with a complex subalgebra of $\mathcal{K}\left(M,-e^{i \hbar / 4}\right)$ preserved by the involution ${ }^{-}$. 
For any integer $k \geqslant 2$, the topological quantum field theories (TQFT) coming from Chern-Simons theory with gauge group $\mathrm{SU}(2)$ provides a complex Hermitian space $V_{k}(\Sigma)$ of finite dimension. In the geometric framework, the integer $k-2$ is called the level while in the combinatorial framework constructed in [BHMV], the authors use the parameter $p=2 k$. Following them, one has a natural algebra morphism

$$
\mathcal{K}\left(M, \zeta_{k}\right) \rightarrow \operatorname{End}\left(V_{k}(\Sigma)\right)
$$

where $\zeta_{k}=-e^{i \pi / 2 k}$ and $\mathcal{K}\left(M, \zeta_{k}\right):=\mathcal{K}(M, A) \otimes_{A=\zeta_{k}} \mathbb{C}$.

For any $f \in \mathcal{K}(M, A)$ and any $k \geqslant 2$, we denote by $\mathrm{Op}_{k}(f)$ the corresponding operator of $V_{k}(\Sigma)$.

Theorem 1.4. For any $f, g \in \mathcal{K}(M, A)$ one has

$$
\mathrm{Op}_{k}(f * g)=\mathrm{Op}_{k}(f) \mathrm{Op}_{k}(g) \text { and } \mathrm{Op}_{k}(\bar{f})=\mathrm{Op}_{k}(f)^{*} \text {, }
$$

and as $k \rightarrow \infty$,

$$
\operatorname{tr}\left(\mathrm{Op}_{k}(f)\right)=\left(\frac{k}{4 \pi^{2}}\right)^{n} \int_{\mathcal{M}^{s}} \sigma(f) \mu+O\left(k^{n-1}\right)
$$

where $n$ is half the dimension of $\mathcal{M}^{s}$ and $\mu$ is the Liouville measure.

First part follows from the general properties of TQFT. The estimation of the trace has been proved in $[\mathrm{MN}]$. A factor $4 \pi^{2}$ appears due to the different normalization of the symplectic form. This estimation gives some information on the asymptotic behavior of $\mathrm{Op}_{k}(f)$.

Define the normalized Hilbert-Schmidt norm of $T \in \operatorname{End}\left(V_{k}(\Sigma)\right)$ by

$$
\|T\|_{\mathrm{HS}}:=\left(\operatorname{dim}\left(V_{k}(\Sigma)\right)\right)^{-1} \operatorname{tr}\left(T T^{*}\right) .
$$

Then by Theorem 1.3 and 1.4 , for any $f \in \mathcal{K}(M, A)$, the Hilbert-Schmidt norm of $\mathrm{Op}_{k}(f)$ is estimated by the $L^{2}$ norm of $g=\sigma(f)$ :

$$
\left\|\mathrm{Op}_{k}(f)\right\|_{H S}^{2}=(\operatorname{Vol}(\mathcal{M}))^{-1} \int_{\mathcal{M}^{s}}|g|^{2} \mu+O\left(k^{-1}\right) .
$$

The space of sequences $\left(T_{k}\right) \in \prod_{k \geqslant 2} \operatorname{End}\left(V_{k}(\Sigma)\right)$ with a bounded Hilbert-Schmidt norm has a natural filtration $O(0) \supset O(1) \supset O(2) \supset \cdots$ where $\left(T_{k}\right)$ is in $O(\ell)$ if $\left\|T_{k}\right\|_{\mathrm{HS}} \leqslant C k^{-\ell}$ for some $C$. It follows from Theorems 1.3 and 1.4 that this filtration corresponds to the formal one:

$$
\left(\mathrm{Op}_{k}(f)\right)_{k} \in O(\ell) \Longleftrightarrow f \in \hbar^{\ell} \mathcal{K}\left(M,-e^{i \hbar / 4}\right),
$$

for any $f \in \mathcal{K}(M, A)$. In particular, if $f \neq 0, \mathrm{Op}_{k}(f)$ does not vanish when $k$ is sufficiently large. 
Using this last observation, one may deduce that the action on $V_{k}(\Sigma)$ of an homeomorphism $\varphi$ of $\Sigma$ provided by TQFT is asymptotically non trivial as soon as the action of this homeomorphism on the Kauffman module is non trivial. This occurs only if $\varphi$ is isotopic to the identity, the elliptic or the hyperelliptic involution, cf. $[\mathrm{MN}]$ for details.

\section{Dehn coordinates}

Given $\Sigma$ an oriented compact surface with boundary, we will call multicurve a submanifold of $\Sigma$ which does not meet the boundary and has no component bounding a disc. The Dehn theorem classifies isotopy classes of multicurves by decomposing them into simple pieces: in these simple pieces, we will allow multicurves to meet the boundary transversally as we explain below.

Let $w$ be the arc of points in $S^{1}$ whose angles are in $[-\pi / 8, \pi / 8]$. For every $m \in \mathbb{N}$ we fix a subset $W_{m}$ of cardinality $m$ in $w$ invariant by complex conjugation. Let $A=S^{1} \times[0,1]$ be an annulus and introduce a family $A(m, t)$ of multicurves of $A$ indexed by $\mathbb{N} \times \mathbb{Z}$. If $m$ is positive, $A(m, t)$ is the multicurve in $A$ (unique up to isotopy) whose projection on $[0,1]$ is a submersion, which intersects each boundary circle in $W_{m}$ and whose algebraic intersection with the curve $\{-1\} \times[0,1]$ equals $t$ (oriented from 0 to 1 ). An example is shown in the left hand side of Figure 2. In the case where $m=0, A(m, t)$ consists of $t$ parallel copies of the boundary.

Let $T$ be the surface $\left\{z \in \mathbb{C}\right.$, such that $|z| \leq 1$ and $\left.\left|z \pm \frac{1}{2}\right|>\frac{1}{4}\right\}$. Choose identifications of the boundary circles of $T$ with $S^{1}$ such that $1 \in S^{1}$ is identified respectively with $p_{1}=\frac{1}{4}, p_{2}=\frac{-1}{4}$ and $p_{3}=i$. We call $T$ the standard pair of pants (or trinion). Let $m_{1}, m_{2}, m_{3}$ be three non-negative integers with even sum. Consider a multicurve as in the right hand side of Figure 2 which intersects the boundary circles in $W_{m_{1}}, W_{m_{2}}, W_{m_{3}}$ via the identifications with $S^{1}$. Denote it by $C\left(m_{1}, m_{2}, m_{3}\right)$.

Consider now a general surface $\Sigma$ with negative Euler characteristic. From the classification of surfaces, it follows that $\Sigma$ can be obtained by gluing trinions on boundary components. We will call pant decomposition of $\Sigma$ a homeomorphism

$$
\Phi: \Sigma \rightarrow\left(\bigcup_{i \in I} T_{i} \cup \bigcup_{j \in J} A_{j}\right) / \varphi
$$

where the $T_{i}$ 's are copies of $T$, the $A_{j}$ 's are copies of $A$ and $\varphi$ is a collection of homeomorphisms reversing the orientation between boundary components of the $T_{i}$ 's and the $A_{j}$ 's. We ask that these homeomorphisms reduce to either the identity or the complex conjugation via the identifications of the boundary components with $S^{1}$, so that they preserve the subsets $W_{n}$. We also ask that each boundary component of a copy of $T$ is glued to some copy of $A$. In that way, the components of the boundary of $\Sigma$ correspond to some copies of $A$. 

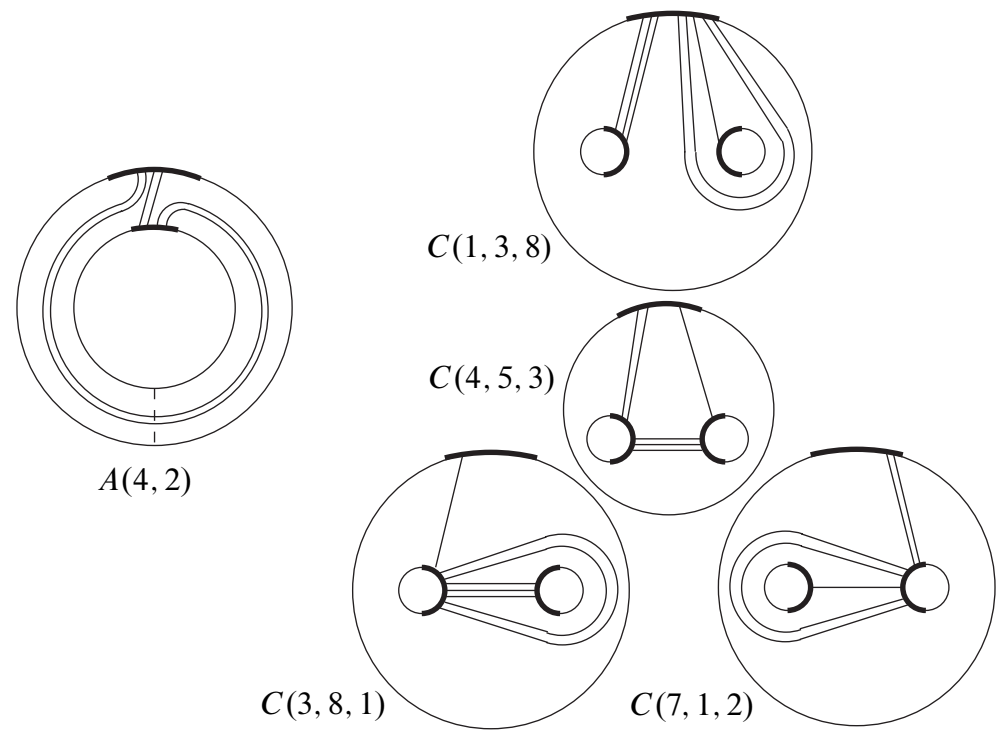

Figure 2. Examples of elementary multicurves.

We associate to a pants decomposition of $\Sigma$ the following graph $G$. Its vertex set is $I \cup \pi_{0}(\partial \Sigma)$. Edges are indexed by $J$ in such a way that the edge associated to the annulus $A_{j}$ connects the copies of $T$ or the boundary component of $\Sigma$ to which it is attached. It happens that the vertices in $I$ are trivalent and the vertices in $\pi_{0}(\partial \Sigma)$ are univalent. An edge is said internal if it connects trivalent vertices, otherwise it is said external.

Consider now a surface with a pants decomposition with graph $G$. We will call Dehn parameter a pair $(m, t)$ where $m$ is a map from the edges of $G$ to $\mathbb{N}$ and $t$ is a map from the edges of $G$ to $\mathbb{Z}$ satisfying the following conditions:

- if $j_{1}, j_{2}, j_{3}$ are three edges incoming to the same vertex, then $m_{j_{1}}+m_{j_{2}}+m_{j_{3}}$ is even.

- for all edges $j$, if $m_{j}=0$ then $t_{j} \geq 0$.

- all external edges $j$ satisfy $m_{j}=0$.

Given such a Dehn parameter, one can construct a multicurve on $\Sigma$ by gluing elementary multicurves in the following way: define $C(m, t)$ as the union

$$
\bigcup_{i} C\left(m_{1}^{i}, m_{2}^{i}, m_{3}^{i}\right) \cup \bigcup_{j} A\left(m_{j}, t_{j}\right) / \varphi
$$

In this expression, $m_{1}^{i}, m_{2}^{i}, m_{3}^{i}$ are the values of $m$ at edges adjacent to $i$.

The classification theorem of Dehn is the following. 
Theorem 2.1. Let $\Sigma$ be a surface with pants decomposition. The map sending a Dehn parameter $(m, t)$ to the multicurve $C(m, t)$ is a bijection between the set of Dehn parameters and the set of isotopy classes of multicurves in $\Sigma$.

We refer to [FLP] for the proof. In the sequel we only need to know that each multicurve of $\Sigma$ is isotopic to some $C(m, t)$. The fact that $C(m, t)$ is isotopic to $C\left(m^{\prime}, t^{\prime}\right)$ only if $m=m^{\prime}$ and $t=t^{\prime}$ is a consequence of our results.

\section{Moduli spaces and torus actions}

Let $\Sigma$ be an oriented compact surface with boundary. Let $\mathcal{M}(\Sigma)$ be the set of isomorphism classes of pairs $(E, \theta)$ where $E$ is a flat Hermitian bundle of rank 2 over $\Sigma$ and $\theta$ is a volume section, that is a flat unitary section of $\wedge^{2} E$. An isomorphism between two pairs is an isomorphism of flat Hermitian bundles commuting with the volume sections. The holonomy representation of the fundamental group $\pi$ induces an isomorphism

$$
\mathcal{M}(\Sigma) \rightarrow \operatorname{Hom}(\pi, \mathrm{SU}(2)) / \mathrm{SU}(2) .
$$

To any pants decomposition is associated a torus action on a dense open subset of this moduli space together with a set of invariant functions which separate the orbits.

Let $C$ be a simple curve (embedded circle) of $\Sigma$. Choose an orientation of $C$ and a base point $x \in C$. Then for any $(E, \theta) \in \mathcal{M}(\Sigma)$, the holonomy at $x$ of $\left.E\right|_{C}$ is a unitary automorphism $g_{x}$ of $E_{x}$ preserving $\theta_{x}$. So the trace of $g_{x}$ belongs to [-2,2]. We set

$$
a_{C}(E, \theta):=\arccos \left(\frac{1}{2} \operatorname{tr}\left(g_{x}\right)\right) \in[0, \pi] .
$$

This defines a function $a_{C}$ on the moduli space $\mathcal{M}(\Sigma)$ which does not depend on the choices of the base point and the orientation, these functions were introduced by Goldman in [Gol].

Let us introduce a circle action on the subset of $\mathcal{M}(\Sigma)$ consisting of bundles with a non-central holonomy along $C$. Let $t \in \mathbb{T}:=\mathbb{R} / \mathbb{Z}$. To define $t$. $(E, \theta)$, we assume that $C$ is oriented. Then $\left.E\right|_{C}$ is isomorphic to $E^{+} \oplus E^{-}$: the holonomy along $C$ preserves this decomposition and acts on $E^{+}$by $\exp \left(i a_{C}(E)\right)$ and on $E^{-}$ by $\exp \left(-i a_{C}(E)\right)$. Let $R_{t}$ be the automorphism of $\left.E\right|_{C}$ which acts on $E^{ \pm}$by multiplication by $\exp ( \pm 2 i \pi t)$.

Next consider the surface $\Sigma^{\prime}$ obtained from $\Sigma$ by cutting out $C$. Denote by $\pi$ the projection from $\Sigma^{\prime}$ to $\Sigma$. Let $C_{+}$and $C_{-}$be the boundary components of $\Sigma^{\prime}$ such that $\pi$ restricts to diffeomorphisms $\pi_{ \pm}: C_{ \pm} \rightarrow C$ and which respectively preserves and reverses the orientation. Then $t .(E, \theta)$ is the quotient of $\pi^{*} E$ under the identification

$$
u \sim \pi_{-}^{*} \cdot R_{t} \cdot\left(\pi_{+}^{*}\right)^{-1}(u),\left.\quad u \in \pi^{*} E\right|_{C_{+}}=\pi_{+}^{*}\left(\left.E\right|_{C}\right)
$$

This definition does not depend on the orientation of $C$. 
Let $\mathscr{C}$ be a set of simple disjoint curves. Then the corresponding actions commute and we obtain an action of the torus $\mathbb{T}^{\mathscr{C}}$ on the set $\mathcal{M}^{0}(\Sigma)$ of bundles with noncentral holonomy along any curve of $\mathscr{C}$. Assume that the curves of $\mathscr{C}$ cut $\Sigma$ into trinions. As in Section 2 we define a graph $G$ whose edges are naturally indexed by $J:=\mathscr{C} \cup \pi_{0}(\partial \Sigma)$. Introduce the map

$$
a: \mathcal{M}(\Sigma) \rightarrow \mathbb{R}^{J}
$$

whose coordinates are the $a_{C}$ 's.

Theorem 3.1. The image of $a$ is the polyhedron $\Delta$ consisting of the $\left(\alpha_{j}\right) \in \mathbb{R}^{J}$ such that for any trivalent vertex $v$ of $G$

$$
\left|\alpha_{i}-\alpha_{j}\right| \leqslant \alpha_{k} \leqslant \min \left(\alpha_{i}+\alpha_{j}, 2 \pi-\left(\alpha_{i}+\alpha_{j}\right)\right)
$$

if $i, j$ and $k$ are the edges incident to $v$. The fibers of the restriction of a to $\mathcal{M}^{0}(\Sigma)$ are the orbits of the action of $\mathbb{T}^{\mathscr{C}}$. Furthermore the action is locally free on the preimage of the interior of $\Delta$.

This theorem has been proved in [JW1] under the assumption that $\Sigma$ has no boundary. There is no difficulty to generalize to the case where $\partial \Sigma \neq \emptyset$. One shows first the result for a pair of pants $\Sigma$, cf. Proposition 3.1 of [JW1]. The general case follows by analyzing how one can paste flat $\mathrm{SU}(2)$ bundles on the trinions cut out by $\mathscr{C}$ to a global bundle on $\Sigma$.

When $\Sigma$ has no boundary, the open set of irreducible classes $(E, \theta)$ is naturally a symplectic manifold, as we already mentioned it in Section 1.3. Then for any simple curve $C$, the function $a_{C}(E)$ is a moment of the circle action corresponding to $C$, that is its symplectic gradient generates the circle action, see [JW1], Proposition 5.4.

It is easily seen that $\Delta$ has a non-empty interior. Furthermore the restrictions to $\operatorname{Int}(\Delta)$ of the functions

$$
\exp \left(i \sum_{j \in J} \ell_{j} a_{j}\right), \quad\left(\ell_{j}\right) \in \mathbb{Z}^{J},
$$

are linearly independent where $a_{j}$ denotes the $j$-th component of $a$. This will be used in the proof of Theorem 5.3. The fact that $\Delta$ has a non-empty interior will also ensure that some Fourier coefficients do not vanish, cf. Lemma 4.4.

\section{Fourier decomposition}

We are concerned in this part on the Fourier decomposition on some trace functions defined on the moduli spaces discussed above. Consider a disjoint union $\Gamma$ of unoriented circles and a map $h: \Gamma \rightarrow \Sigma$. Then define the following function on $\mathcal{M}(\Sigma)$ :

$$
T_{h}(E, \theta)=\prod_{\gamma \in \pi_{0}(\Gamma)}\left(-\operatorname{tr}\left(g_{\gamma}\right)\right)
$$


where $g_{\gamma}$ is the holonomy of $E$ along $h(\gamma)$. Let $\mathscr{C}$ be a finite family of disjoint simple closed curves. Then for any $k \in \mathbb{Z}^{\mathscr{C}}$, the $k$-th isotype of $T_{h}$ for the action of the torus $\mathbb{T}^{\mathscr{C}}$ is the function

$$
\Pi_{k}\left(T_{h}\right)(x)=\int_{\mathbb{T}^{\mathscr{C}}} T_{h}(t . x) e^{-2 \pi i\langle t, k\rangle} d t .
$$

The first step in the computation of $\Pi_{k}\left(T_{h}\right)$ is to extend the definition of $T_{h}(E, \theta)$ to the case where $\Gamma$ is a disjoint union of circles and closed intervals.

4.1. Generalized holonomy trace. Let us start with an algebraic preliminary. Consider a finite family $\left(V_{i}\right)_{i \in I}$ of vector spaces. Let us define the tensor product $\bigotimes_{i \in I} V_{i}$ assuming that the $V_{i}$ are odd superspaces. First, for any bijections $\sigma$ and $\sigma^{\prime}$ from $\{1, \ldots, n\}$ to $I$, one has a commutation map

$$
c_{\sigma, \sigma^{\prime}}: V_{\sigma(1)} \otimes \cdots \otimes V_{\sigma(n)} \rightarrow V_{\sigma^{\prime}(1)} \otimes \cdots \otimes V_{\sigma^{\prime}(n)}
$$

sending $v_{1} \otimes \cdots \otimes v_{n}$ to $(-1)^{\epsilon(\alpha)} v_{\alpha(1)} \otimes \cdots \otimes v_{\alpha(n)}$ where $\alpha=\sigma^{\prime} \circ \sigma^{-1}$. Since $c_{\sigma^{\prime}, \sigma^{\prime \prime}} \circ c_{\sigma, \sigma^{\prime}}=c_{\sigma, \sigma^{\prime \prime}}$, one can identify coherently these tensor products by taking the projective limit of this system, which defines the unordered tensor product. More precisely, we define $\otimes_{i \in I} V_{i}$ as the subspace of the product

$$
\prod_{\sigma \in \operatorname{bij}(\{1, \ldots, n\}, I)}\left(V_{\sigma(1)} \otimes \cdots \otimes V_{\sigma(n)}\right)
$$

consisting of the families $\left(v_{\sigma}\right)$ such that $c_{\sigma, \sigma^{\prime}}\left(v_{\sigma}\right)=v_{\sigma^{\prime}}$.

Let $E \rightarrow \Sigma$ be a flat vector bundle of rank 2 and $\theta$ a non-vanishing flat section of $\wedge^{2} E$. Observe that each fiber $E_{x}$ has a complex symplectic product $\omega_{x}$ defined in such a way that for all $u, v \in E_{x}$,

$$
u \wedge v=\theta_{x} \Rightarrow \omega_{x}(u, v)=1 .
$$

Consider a 1-dimensional compact manifold $\Gamma$ and a continuous function $h: \Gamma \rightarrow$ $\Sigma$. We will define the generalized holonomy trace of $h$ as a vector

$$
T_{h}(E, \theta) \in \bigotimes_{p \in \partial \Gamma} E_{h(p)}
$$

If $\Gamma$ is an interval, we choose an orientation of it and denote by $p$ and $q$ the source and target point of $\Gamma$. Then the holonomy along $\Gamma$ is an homomorphism

$$
A \in \operatorname{Hom}\left(E_{h(p)}, E_{h(q)}\right) \simeq E_{h(q)} \otimes E_{h(p)}^{*} .
$$

Identifying $E_{h(p)}$ with $E_{h(p)}^{*}$ by the map sending $u$ to $\omega_{h(p)}(u, \cdot)$, we obtain a vector $A_{\mathrm{b}} \in E_{h(q)} \otimes E_{h(p)}$. We set

$$
T_{h}(E, \theta):=A_{\mathrm{b}} .
$$


The important point is that this definition does not depend of the orientation of $\Gamma$ if we use the previous identification between $E_{h(p)} \otimes E_{h(q)}$ and $E_{h(q)} \otimes E_{h(p)}$. Indeed with this identification, we have that $\left(A^{-1}\right)_{b}=A_{b}$. This is easily deduced from the fact that $A$ is a linear symplectomorphism. If $\Gamma$ is a circle, we define $T_{h}(E, \theta)$ as before as the opposite of the trace of the holonomy of $h$ (the super-trace). Finally, if $\Gamma$ has several components $\left(\Gamma_{i}\right)$, we define $T_{h}(E, \theta)$ as the tensor product of the $T_{\left.h\right|_{\Gamma_{i}}}(E, \theta)$.

Now let $h^{\prime}: \Gamma^{\prime} \rightarrow \Sigma$ be a continuous map and $p$ and $q$ be two distinct points in $\partial \Gamma^{\prime}$ such that $h^{\prime}(p)=h^{\prime}(q)$. Then by identifying $p$ with $q$, we get a compact 1 -manifold $\Gamma$ with a map $h: \Gamma \rightarrow \Sigma$. It is easily checked that

$$
T_{h}(E, \theta)=\operatorname{Cont}\left(T_{h^{\prime}}(E, \theta)\right)
$$

where Cont is the contraction

$$
\bigotimes_{r \in \partial \Gamma^{\prime}} E_{h(r)} \longrightarrow \bigotimes_{r \in \partial \Gamma^{\prime} \backslash\{p, q\}} E_{h(r)}
$$

sending $u \otimes v \otimes w$ to $u . \omega(v, w)$ if $w \in E_{h(p)}, v \in E_{h(q)}$ and $u \in \bigotimes_{r \in \partial \Gamma^{\prime} \backslash\{p, q\}} E_{h(r)}$.

4.2. The basic computation. In this part we consider the case of a single simple closed curve $C$ of $\Sigma$ with its associated circle action. Let $\Gamma$ be a finite disjoint union of circles and $h: \Gamma \rightarrow \Sigma$ be a map intersecting $C$ transversally.

Let $\Gamma^{\prime}$ be the compact 1-manifold obtained by cutting out the points $p$ of $\Gamma$ such that $h(p)$ is on $C$ and compactifying. Let $h^{\prime}$ be the obvious map $\Gamma^{\prime} \rightarrow \Sigma$. Then it follows from the considerations of Section 4.1 that $T_{h}(E, \theta)$ is the contraction of the generalized trace holonomy $T_{h^{\prime}}(E, \theta)$ by the linear map

$$
\text { Cont: } \bigotimes_{p \in \partial \Gamma^{\prime}} E_{h^{\prime}(p)} \rightarrow \mathbb{C}
$$

defined as follows. For any $p \in h^{-1}(C)$, let $j_{1}(p)$ and $j_{2}(p)$ be the corresponding boundary points of $\Gamma^{\prime}$ chosen in arbitrary order, so that

$$
\bigotimes_{p \in \partial \Gamma^{\prime}} E_{h^{\prime}(p)}=\bigotimes_{p \in h^{-1}(C)} E_{h(p)}^{\otimes\left\{j_{1}(p), j_{2}(p)\right\}} .
$$

Then Cont is the map sending $\bigotimes_{p \in h^{-1}(C)}\left(v_{p, 1} \otimes v_{p, 2}\right)$ to $\prod_{p \in h^{-1}(C)} \omega_{h(p)}\left(v_{p, 1}, v_{p, 2}\right)$. We will compute $\Pi_{k}\left(T_{h}\right)$ in terms of these generalized holonomy traces. To do this we introduce another contraction map from $\bigotimes_{p \in \partial \Gamma^{\prime}} E_{h^{\prime}(p)}$ to $\mathbb{C}$.

Let $p \in h^{-1}(C)$ and let us orientate $C$ in such a way that the tangent vector of $\Gamma$ oriented from $j_{1}(p)$ to $j_{2}(p)$ followed by the oriented tangent vector of $C$ form a direct basis in $\Sigma$. Assume that the holonomy of $(E, \theta)$ along $C$ is not central. Choose two unitary eigenvectors $e_{ \pm}^{p} \in E_{h(p)}$ of the holonomy along $C$ with corresponding 
eigenvalues $\exp \left( \pm i a_{C}(E)\right)$ and such that $\theta_{h(p)}=e_{+}^{p} \wedge e_{-}^{p}$. Then consider the linear maps

$$
\mathrm{Cont}_{ \pm}^{p}: E_{h(p)} \otimes E_{h(p)} \rightarrow \mathbb{C}, \quad v_{1} \otimes v_{2} \rightarrow\left\langle v_{1}, e_{ \pm}^{p}\right\rangle\left\langle v_{2}, e_{\mp}^{p}\right\rangle
$$

where the bracket denotes the scalar product. Exchanging $j_{1}(p)$ and $j_{2}(p)$ and defining the same application, we obtain a map $\widetilde{\operatorname{Cont}}_{ \pm}^{p}$ satisfying

$$
\operatorname{Cont}_{ \pm}^{p}\left(v_{1} \otimes v_{2}\right)=-\widetilde{\operatorname{Cont}}_{ \pm}^{p}\left(v_{2} \otimes v_{1}\right) .
$$

So we have a well-defined contraction $E_{h(p)}^{\left\{j_{1}(p), j_{2}(p)\right\}} \rightarrow \mathbb{C}$. Finally we set,

$\mathrm{Cont}_{ \pm}^{C}: \bigotimes_{p \in \partial \Gamma^{\prime}} E_{h^{\prime}(p)} \rightarrow \mathbb{C}, \bigotimes_{p \in h^{-1}(C)}\left(v_{p, 1} \otimes v_{p, 2}\right) \rightarrow \prod_{p \in h^{-1}(C)} \operatorname{Cont}_{ \pm}^{p}\left(v_{p, 1} \otimes v_{p, 2}\right)$

where we used the decomposition (4) into a product of even superspaces. If $C$ does not intersect $h$, Cont ${ }_{ \pm}^{C}$ is the identity map.

Lemma 4.1. Let $k \in \mathbb{N}$. If $k$ is bigger than the cardinality of $h^{-1}(C)$, then $\Pi_{k}\left(T_{h}\right)$ and $\Pi_{-k}\left(T_{h}\right)$ vanish. If $k$ is equal to the cardinality of $h^{-1}(C)$, then

$$
\Pi_{ \pm k}\left(T_{h}\right)(E, \theta)=\mathrm{Cont}_{ \pm}^{C}\left(T_{h^{\prime}}(E, \theta)\right) .
$$

Proof. Assume first that $h^{-1}(C)$ is reduced to a point. Then the proof is based on the simple observation that

$$
T_{h}(t .(E, \theta))=\operatorname{Cont}_{t}\left(T_{h^{\prime}}(E, \theta)\right) \quad \text { for all } t \in \mathbb{T}
$$

where Cont $t_{t}$ is the linear map from $E_{h(p)}^{\otimes\left\{j_{1}(p), j_{2}(p)\right\}}$ to $\mathbb{C}$ given by

$$
\operatorname{Cont}_{t}\left(v_{1} \otimes v_{2}\right)=\omega_{h(p)}\left(R_{t} v_{1}, v_{2}\right)
$$

with $R_{t}$ the automorphism of $E_{h(p)}$ entering in the definition of the circle action. Next a straightforward computation leads to

$$
\omega_{h(p)}\left(R_{t} v_{1}, v_{2}\right)=e^{2 i \pi t}\left\langle v_{1}, e_{+}^{p}\right\rangle\left\langle v_{2}, e_{-}^{p}\right\rangle+e^{-2 i \pi t}\left\langle v_{1}, e_{-}^{p}\right\rangle\left\langle v_{2}, e_{+}^{p}\right\rangle
$$

which proves the result, taking into account that the Hermitian product is $\mathbb{C}$-linear on the left. In the case where $h^{-1}(C)$ consists of several points, (5) is still satisfied with the contraction

$$
\operatorname{Cont}_{t}^{C}\left(\bigotimes_{p \in h^{-1}(C)}\left(v_{p, 1} \otimes v_{p, 2}\right)\right)=\prod_{p \in h^{-1}(C)} \omega_{h(p)}\left(R_{t} v_{p, 1}, v_{p, 2}\right) .
$$

Replacing each term in the product by the previous formula leads to the result. 
Consider now a finite set $\mathscr{C}$ of disjoint simple closed curves. One can generalize the previous lemma with the action of $\mathbb{T}^{\mathscr{C}}$. Assume that $h: \Gamma \rightarrow \Sigma$ intersects each $C \in \mathscr{C}$ transversally. Consider the manifold $\Gamma^{\prime}$ and the map $h^{\prime}: \Gamma^{\prime} \rightarrow \Sigma$ obtained from $h: \Gamma \rightarrow \Sigma$ by cutting out the $p$ 's such that $h(p) \in C$ for some $C \in \mathscr{C}$. Then one has the following decomposition into a tensor product of even superspaces

$$
\bigotimes_{p \in \partial \Gamma^{\prime}} E_{h^{\prime}(p)}=\bigotimes_{C \in \mathscr{C}}\left(\bigotimes_{p \in \partial \Gamma^{\prime} / h^{\prime}(p) \in C} E_{h^{\prime}(p)}\right)
$$

So for any $\varepsilon \in\{+,-\}^{\mathscr{C}}$ we can define a linear map

$$
\operatorname{Cont}_{\varepsilon}^{\mathscr{C}}=\prod_{C \in \mathscr{C}} \operatorname{Cont}_{\varepsilon_{C}}^{C}: \bigotimes_{p \in \partial \Gamma^{\prime}} E_{h^{\prime}(p)} \rightarrow \mathbb{C}
$$

where the Cont ${ }_{ \pm}^{C}$ are the previously defined contractions.

Lemma 4.2. Let $k \in \mathbb{Z}^{\mathscr{C}}$. If there exists $C \in \mathscr{C}$ such that $\left|k_{C}\right|>\left|h^{-1}(C)\right|$, then $\Pi_{k}\left(T_{h}\right)$ vanishes. If for any $C \in \mathscr{C}$ we have $\left|k_{C}\right|=\left|h^{-1}(C)\right|$, then

$$
\Pi_{k}\left(T_{h}(E, \theta)=\operatorname{Cont}_{\varepsilon}^{\mathscr{C}}\left(T_{h^{\prime}}(E, \theta)\right)\right.
$$

with $\varepsilon_{C}=\operatorname{sgn}\left(k_{C}\right)$ for any $C \in \mathscr{C}$.

Proof. The proof is the same as the one of Lemma 4.1. Indeed equation (5) is still satisfied with the contraction $\operatorname{Cont}_{t}^{\mathscr{C}}$ defined as the product of the $\operatorname{Cont}_{t_{C}}^{C}$ given in (6) where $C$ runs over $\mathscr{C}$.

4.3. Fractional Dehn twist. Consider again a curve $C$ on $\Sigma$ and a map $h: \Gamma \rightarrow \Sigma$ which intersects $C$ transversally in $k$ points. One defines the fractional Dehn twist of $h$ along $C$ of order $\ell / k$ by cutting $\Gamma$ along $C$ and connecting each strand with the $\ell$-th consecutive one.

Formally, it is defined as follows. Let us orient arbitrarily $C$. Let $\Gamma^{\prime}$ be the 1manifold obtained by cutting out from $\Gamma$ the points $p$ such that $h(p)$ is on $C$ and compactifying. For any $p \in h^{-1}(C)$, denote by $j_{1}(p)$ and $j_{2}(p)$ the corresponding boundary point of $\Gamma^{\prime}$ such that $C$ goes left when seen from $j_{1}(p)$. This is the same convention as in Section 4.2, except that the orientation of the curve $C$ does not depend on $p$. The orientation of $C$ provides $P=h^{-1}(C)$ with a cyclic order, so $\mathbb{Z} / k \mathbb{Z}$ acts on $P$. One sets

$$
\Gamma_{\ell / k}=\Gamma^{\prime} \cup([0,1] \times P) / \sim
$$

where we identify $j_{1}(p)$ with $(0, p)$ and $j_{2}(p+\ell)$ with $(1, p)$ for any $p \in P$.

Choose a parametrization $\alpha: S^{1} \rightarrow C$ respecting the orientation such that $P$ corresponds to the set of $k$-th roots of unity. We define the map $h_{\ell / k}: \Gamma_{\ell / k} \rightarrow \Sigma$ as being equal to $h$ on $\Gamma^{\prime}$ and such that for all $(t, p) \in[0,1] \times P$ one has

$$
h_{\ell / k}(t, p)=\alpha\left(\exp \left(\frac{2 i \pi \ell}{k} t\right) \cdot \alpha^{-1}(h(p))\right) .
$$


The manifold $\Gamma_{\ell / k}$ and the map $h_{\ell / k}$ do not depend up to homotopy on the orientation of $C$ nor on the parametrization $\alpha$.

Lemma 4.3. One has $\Pi_{ \pm k}\left(T_{h_{\ell / k}}\right)=(-1)^{\ell(k-1)} e^{ \pm i \ell a_{C}} \Pi_{ \pm k}\left(T_{h}\right)$.

Proof. This is an easy application of the Lemma 4.1 using the explicit description of the fractional Dehn twist above. More precisely, denote by $H_{\ell / k}$ the restriction of $h_{\ell / k}$ to $[0,1] \times P$ and choose a parametrization $\alpha: S^{1} \rightarrow C$ as above. Given a bundle $E$ in $\mathcal{M}(\Sigma)$ one can suppose that it is trivialized on $P$ in such a way that the holonomy from $p$ to $p+1$ is

$$
\left[\begin{array}{cc}
\exp \left(2 i \pi a_{C} / k\right) & 0 \\
0 & \exp \left(-2 i \pi a_{C} / k\right)
\end{array}\right] .
$$

Then $H_{\ell / k}$ and $H_{0 / k}$ differ by the factor $\exp \left(2 i \pi a_{C} / k\right)^{k \ell}$ and the sign of the permutation sending $x$ to $x+\ell$ in $\mathbb{Z} / k \mathbb{Z}$.

4.4. Non vanishing of some isotypes. Consider a pants decomposition of $\Sigma$ in the sense of Section 2 with graph $G$. Let $S^{1} \times\{1 / 2\}$ be the core of the standard annulus $S^{1} \times[0,1]$. For each internal edges of $G$, we consider the core of the corresponding annulus and the circle action it generates. This defines a torus action of $\mathbb{T}^{\mathscr{C}}$ where $\mathscr{C}$ is the set of internal edges.

Consider the multicurve $C(m, t)$ with Dehn coordinates $(m, t)$. By the first part of Lemma 4.2, the $k$-th isotype of $T_{C(m, t)}$ vanishes if $\left|k_{j}\right|>m_{j}$ for some internal edge $j$.

Lemma 4.4. Let $k \in \mathbb{Z}^{\mathscr{C}}$ be such that $\left|k_{j}\right|=m_{j}$ for all $j \in \mathscr{C}$. Then the function $\Pi_{k}\left(T_{C(m, t)}\right)$ is nowhere 0 on $a^{-1}(\operatorname{Int}(\Delta))$, where a is the map introduced in Section 3 and $\Delta$ is the image of $a$.

Proof. By Lemma 4.3, one may assume that $t$ vanishes. We computed $\Pi_{k}\left(T_{C(m, 0)}\right)$ in Lemma 4.2 in terms of a contraction $\operatorname{Cont}_{\varepsilon}^{\mathscr{C}}$ defined as a product over the boundary points of $\Gamma^{\prime}$ as follows.

For any curve $C$ in $\mathscr{C}$, any $p$ of $C$ and any choice of orientation of $C$, one considers a unitary eigenvector $e$ of $E_{p}$ for the holonomy along $C$ whose eigenvalue has positive imaginary part. To any point $p$ in $\partial \Gamma^{\prime}$ such that $h^{\prime}(p) \in C$, one associates the vector $e_{+}^{p}$ (resp. $e_{-}^{p}$ ) corresponding to the orientation of $C$ going to the left from $p$ (resp. to the right). For any $p \in \partial \Gamma$, denote by $\varepsilon(k, p)$ the sign of $k_{j}$ where $h(p)$ belongs to $j \in \mathscr{C}$. Then

$$
\operatorname{Cont}_{\varepsilon}^{\mathscr{C}}\left(\bigotimes_{p \in \partial \Gamma^{\prime}} v_{p}\right)= \pm \prod_{p \in \partial \Gamma^{\prime}}\left\langle v_{p}, e_{\varepsilon(k, p)}^{p}\right\rangle .
$$

Here the equality is only true modulo a plus or minus sign because we have not chosen an order of $\partial \Gamma^{\prime}$, so the sign of the left hand side is not defined. 
Since $T_{h^{\prime}}(E, \theta)$ is the tensor product of the $T_{\left.h^{\prime}\right|_{\gamma}}(E, \theta)$ where $\gamma$ runs overs the components of $\Gamma^{\prime}$, we have by Lemma 4.2

$$
\Pi_{k}\left(T_{h}\right)(E, \theta)= \pm \prod_{\gamma \in \pi_{0}\left(\Gamma^{\prime}\right)} \operatorname{Cont}^{\gamma}\left(T_{\left.h^{\prime}\right|_{\gamma}}(E, \theta)\right)
$$

with $\operatorname{Cont}^{\gamma}\left(v_{p} \otimes v_{q}\right)=\left\langle v_{p}, e_{\varepsilon(k, p)}^{p}\right\rangle\left\langle v_{q}, e_{\varepsilon(k, q)}^{q}\right\rangle$ if the source and target of $\gamma$ are $p$ and $q$.

To prove the lemma, it suffices to show that the factors $\operatorname{Cont}^{\gamma}\left(T_{\left.h^{\prime}\right|_{\gamma}}(E, \theta)\right)$ do not vanish when $a(E, \theta)$ belongs to the interior of $\Delta$. There are two cases to consider, according to whether the endpoints of $\gamma$ belong to different boundary curves of the pair of pants or the same one, cf. for example $C(1,1,0)$ and $C(2,0,0)$.

In the first case, consider the pair of pants containing $\gamma$ and let $j_{1}, j_{2}$ and $j_{3}$ be the bounding curves with the orientation induced by the pair of pants. Assume that the endpoints $p$ and $q$ of $\gamma$ belongs to $j_{1}$ and $j_{2}$ respectively. If $\operatorname{Cont}^{\gamma}\left(T_{\left.h^{\prime}\right|_{\gamma}}(E, \theta)\right)$ vanishes, one deduces from the definition of $T_{\left.h^{\prime}\right|_{\gamma}}(E, \theta)$ that the holonomy along the loop $\left.h^{\prime}\right|_{\gamma}$ sends the decomposition $\mathbb{C} e_{+}^{p} \oplus \mathbb{C} e_{-}^{p}$ to the decomposition $\mathbb{C} e_{+}^{q} \oplus \mathbb{C} e_{-}^{q}$, permuting possibly the summands. Choosing $p$ as a base point, one obtains that the holonomies along $j_{1}$ and the concatenation $\gamma^{-1} j_{2} \gamma$ commute. Observe furthermore that $j_{3}^{-1}$ is freely isotopic to $\gamma^{-1} j_{2} \gamma j_{1}$. It follows that for some $\varepsilon_{i} \in\{1,-1\}$, the three eigenvalues $\exp \left(\varepsilon_{1} i a_{j_{1}}(E)\right), \exp \left(\varepsilon_{2} i a_{j_{2}}(E)\right), \exp \left(\varepsilon_{3} i a_{j_{3}}(E)\right)$ have product equal to 1 . This implies that

$$
\varepsilon_{1} a_{j_{1}}(E)+\varepsilon_{2} a_{j_{2}}(E)+\varepsilon_{3} a_{j_{3}}(E) \equiv 0 \bmod 2 \pi \mathbb{Z}
$$

This formula is only satisfied if $a(E, \theta)$ belongs to the boundary of $\Delta$.

In the second case, $\left.h^{\prime}\right|_{\gamma}$ connects two points $p$ and $q$ of the same boundary circle $j_{1}$ by going around another boundary circle $j_{2}$. Since the neighborhoods of the endpoints of $\gamma$ are on the same side of $j_{1}$, the eigenvalues of $e_{p}$ and $e_{q}$ are the same. Using this, one shows that if $\operatorname{Cont}^{\gamma}\left(T_{\left.h^{\prime}\right|_{\gamma}}(E, \theta)\right)$ vanishes, then the holonomy along a path joining $j_{1}$ to $j_{2}$ preserves the decomposition into eigenspaces. In other words, we are again in the first case.

\section{Consequences}

5.1. Geometric intersection numbers. The geometric intersection number of two isotopy classes of multicurves $\xi$ and $\eta$ is the minimal number of intersection points of a representant of $\xi$ with a representant of $\eta$.

If $\xi$ has only one connected component, it generates a circle action on $\mathcal{M}(\Sigma)$. One may characterize the geometric intersection number of $\xi$ and $\eta$ in terms of this action and the trace function of $\eta$. 
Theorem 5.1. For any isotopy classes $\xi$ and $\eta$ of a curve and a multicurve respectively, the geometric intersection number of $\xi$ and $\eta$ is the biggest $k$ such that the $k$-th isotype of the trace function of $\eta$ with respect to the circle action generated by $\xi$ does not vanish.

Proof. If $k$ is larger than the geometric intersection number, then the $k$-th isotype vanishes by Lemma 4.1. Conversely, assume that $\xi$ does not bound a disc and is not parallel to a boundary component, otherwise the result is trivially satisfied. Then there is a pants decomposition with a separating curve $C$ representing $\xi$. By Theorem 2.1, $\eta$ may be represented by a Dehn multicurve $C(m, t)$. Then Lemma 4.4 shows that the $k$-isotype of $T_{C(m, t)}$ does not vanish if $k$ is the number of intersection points of $C(m, t)$ with $C$.

The proof shows also that the intersection number is realized when the representative of $\xi$ is a curve of a pants decomposition and the representative of $\eta$ is one of the associated Dehn curves, a well-known result.

The following corollary has been proved in [Gol]. Denote by $\mathcal{M}^{s}(\Sigma)$ the subset of $\mathcal{M}(\Sigma)$ consisting of the irreducible bundles.

Corollary 5.2. Assume that $\Sigma$ is closed so that $\mathcal{M}^{s}(\Sigma)$ is a symplectic manifold. Let $\xi$ be a curve and $\eta$ a multicurve in $\Sigma$. Then the Poisson bracket of the trace functions of $\xi$ and $\eta$ vanishes if and only if $\xi$ and $\eta$ admit non-intersecting representatives.

Proof. It is well known from Goldman's Poisson bracket formula that if $\xi$ and $\eta$ do not intersect, then their Poisson bracket vanishes (see [Gol]). Reciprocally, consider a pants decomposition of $\Sigma$ such that $\xi$ belongs to the cutting curves. It follows from the work of Goldman that $h=\arccos \left(-\frac{1}{2} T_{\xi}\right)$ is a moment of the circle action generated by $\xi$, that is, on $\mathcal{M}^{0}$, the infinitesimal torus action defined by $\xi$ is the Hamiltonian vector field of $h$. Hence, if the Poisson bracket of some function $f: \mathcal{M}^{0} \rightarrow \mathbb{R}$ with $h$ vanishes, then $f$ is constant on the hamiltonian flow of $h$, that is along the circle action associated to $\xi$. Hence with respect to this action, all non-zero Fourier coefficients vanish. By Theorem 5.1 applied to $f=T_{\eta}$, we see that $\eta$ does not intersect $\xi$.

5.2. Independence of trace functions. One can now prove the following theorem:

Theorem 5.3. The functions $T_{\xi}$, where $\xi$ runs over isotopy classes of multicurves in $\Sigma$, are linearly independent.

Since the isomorphism between $\mathcal{M}(\Sigma)$ and $\operatorname{Hom}(\pi, \mathrm{SU}(2)) / \mathrm{SU}(2)$ identifies the function $T_{\xi}$ with the trace function $f_{[\xi], \mathrm{SU}(2)}$, we deduce Theorem 1.1 for the group $\mathrm{SU}(2)$. 
Remark 1. Consider the following map:

$$
j: \operatorname{Hom}(\pi, \operatorname{SU}(2)) / \operatorname{SU}(2) \rightarrow \operatorname{Hom}(\pi, \operatorname{Sl}(2, \mathbb{C})) / \operatorname{Sl}(2, \mathbb{C})
$$

Then $j^{*} f_{\gamma, \mathrm{SI}(2, \mathbb{C})}=f_{\gamma, \mathrm{SU}(2)}$. So the independence of the $f_{\gamma, \mathrm{SU}(2)}$ implies the independence of the $f_{\gamma, \mathrm{Sl}(2, \mathbb{C})}$, which proves Theorem 1.1 for the group $\operatorname{Si}(2, \mathbb{C})$.

Proof. Consider a pants decomposition of $\Sigma$ with associated graph $G$ and the action of the torus $\mathbb{T}^{\mathscr{C}}$ as in Section 4.4. Let $J=\mathscr{C} \cup \pi_{0}(\Sigma)$ be the set of edges of $G$. By Theorem 2.1, one may suppose that $\xi$ runs over the multicurves $C(m, t)$ for admissible indices $m: J \rightarrow \mathbb{N}$ and $t: J \rightarrow \mathbb{Z}$.

Let $\beta$ be a vanishing linear combination of the $T_{\xi}$ 's. Let $\beta=\sum_{m} \beta_{m}$ be its decomposition with respect to the multi-degree $m$. Write

$$
\beta_{m}=\sum_{t} \lambda_{m, t} T_{C(m, t)}
$$

Consider an arbitrary order on $J$ and the corresponding lexicographical order on maps $m$. Let $M$ be the maximal $m$ with a non-vanishing family of coefficients $\left(\lambda_{m, t}\right)_{t}$. By Lemma 4.1, for $m<M$ one has $\Pi_{M}\left(\beta_{m}\right)=0$. Hence $\Pi_{M}\left(\beta_{M}\right)=\Pi_{M}(\beta)=0$. Furthermore, by Lemma 4.3,

$$
\begin{aligned}
\Pi_{M}\left(\beta_{M}\right) & =\sum_{t} \lambda_{M, t} \Pi_{M}\left(T_{C(M, t)}\right) \\
& =\left(\sum_{t} \lambda_{M, t} \prod_{j \in J} \varphi_{M_{j}, t_{j}}\left(a_{j}\right)\right) \Pi_{M}\left(T_{C(M, 0)}\right)
\end{aligned}
$$

where for any integers $k, \ell$ and real $\alpha$,

$$
\varphi_{k, \ell}(\alpha)= \begin{cases}(-1)^{\ell(k-1)} \exp (i \ell \alpha) & \text { if } k \neq 0 \\ (2 \cos (\alpha))^{\ell} & \text { otherwise }\end{cases}
$$

By Lemma 4.4, the function $\Pi_{M}\left(T_{C(M, 0)}\right)$ does not vanish on $a^{-1}($ Int $\Delta)$. Furthermore, by Remark 1, the functions

$$
\prod_{j \in J} \varphi_{M_{j}, t_{j}}\left(a_{j}\right)
$$

where $t$ runs over the maps $J \rightarrow \mathbb{Z}$ such that $(M, t)$ are Dehn coordinates, are linearly independent over $a^{-1}$ (Int $\left.\Delta\right)$. So the coefficients $\lambda_{M, t}$ vanish, contradicting the maximality of $M$. 


\section{The character variety}

Denote by $V$ the vector space $\mathbb{C}^{2}$ and by $G$ the group $\operatorname{Sl}(2, \mathbb{C}) \subset \operatorname{End}(V)$. Let $\pi$ be a finitely generated group. Choosing generators $t_{1}, \ldots, t_{n}$ of $\pi$, we identify the set $\operatorname{Hom}(\pi, G)$ of morphisms with a closed algebraic subset of (End $V)^{n}$ by sending the morphism $\rho$ to $\left(\rho\left(t_{1}\right), \ldots, \rho\left(t_{n}\right)\right)$. This endows $\operatorname{Hom}(\pi, G)$ with a structure of affine variety. Its coordinate ring $\mathbb{C}[\operatorname{Hom}(\pi, G)]$ is the quotient of $\mathbb{C}\left[(\text { End } V)^{n}\right]$ by the ideal of polynomial functions vanishing on $\operatorname{Hom}(\pi, G)$.

The action by conjugation of $G$ on $\operatorname{Hom}(\pi, G)$ is regular. By Hilbert's theorem, the ring of invariant functions is finitely generated. By definition $\mathbb{C}[\operatorname{Hom}(\pi, G)]^{G}$ is the coordinate ring of the character variety.

One may naturally identify this coordinate ring with a subspace of the space of complex valued functions on the quotient $\operatorname{Hom}(\pi, G) / G$. By the following theorem, this subspace is the space $\mathcal{T}(\pi, G)$ of trace functions.

Theorem 6.1. The ring $\mathbb{C}[\operatorname{Hom}(\pi, G)]^{G}$ is generated as a vector space by the functions $\chi_{t}$,

$$
\chi_{t}([\rho])=-\operatorname{tr}(\rho(t)), \quad \rho \in \operatorname{Hom}(\pi, G),
$$

where $t$ runs over $\pi$.

We provide an elementary proof of this well-known result.

Proof. Because of the trace relation (1), it suffices to prove that the ring of regular invariant functions is generated by the $\chi_{t}$ 's as an algebra. Then by averaging the action of the compact subgroup $\mathrm{SU}(2) \subset G$, we get that any invariant regular function is represented by an invariant polynomial function $P \in \mathbb{C}\left[(\text { End } V)^{n}\right]^{G}$.

The symplectic form of $V$ induce an isomorphism between $V$ and $V^{*}$ and consequently an isomorphism between End $V$ and End $V^{*}$. By composing with the transposition map, we define an equivariant isomorphism $a \rightarrow a^{*}$ of End $(V)$. We will prove that $\mathbb{C}\left[(\text { End } V)^{n}\right]^{G}$ is generated as an algebra by the functions

$$
\left(a_{1}, \ldots, a_{n}\right) \rightarrow \operatorname{tr}(w(a))
$$

where $w(a)$ runs over the words in the letters $a_{1}, a_{1}^{*}, \ldots, a_{n}, a_{n}^{*}$. Since the elements of $G$ satisfy $a^{-1}=a^{*}$, this will end the proof.

Let $W=\operatorname{End}(V)$. For any $n$-tuple $\left(d_{1}, \ldots, d_{n}\right)$ of non negative integers, the space of polynomials on $W^{\oplus n}$ which are homogeneous of degree $d_{i}$ on the $i$-th variable is isomorphic with $\bigotimes_{i} \operatorname{Sym}^{d_{i}}\left(W_{i}^{*}\right)$. So

$$
\mathbb{C}\left[W^{\oplus n}\right]=\bigoplus_{d \in \mathbb{N}^{n}} \bigotimes_{i=1, \ldots, n} \operatorname{Sym}^{d_{i}}\left(W^{*}\right) \subset \bigoplus_{d \in \mathbb{N}^{n}} \bigotimes_{i=1, \ldots, n}\left(W^{*}\right)^{\otimes d_{i}}=: E .
$$


A right inverse of this inclusion is the following map from $E$ to $\mathbb{C}\left[W^{\oplus n}\right]$

$$
\bigotimes_{i=1, \ldots, n}\left(\ell_{i}^{1} \otimes \cdots \otimes \ell_{i}^{d_{i}}\right) \rightarrow P\left(a_{1}, \ldots, a_{n}\right)=\prod_{\substack{i=1, \ldots, n ; \\ j=1, \ldots, d_{i}}} \ell_{i}^{j}\left(a_{i}\right)
$$

Observe that the invariant subspace of $E$ is sent onto $\mathbb{C}\left[W^{\oplus n}\right]^{G}$. Using again the identification of $V$ with $V^{*}$, we have that $W \simeq V \otimes V^{*} \simeq V^{*} \otimes V^{*}$. By Lemma 6.2, the invariant subspace of $\left(V^{*}\right)^{\otimes 2 m} \simeq\left(V^{\otimes 2 m}\right)^{*}$ is generated by the maps

$$
x_{1} \otimes \cdots \otimes x_{2 m} \rightarrow \omega\left(x_{\sigma(1)}, x_{\sigma(2)}\right) \ldots \omega\left(x_{\sigma(2 m-1)}, x_{\sigma(2 m)}\right)
$$

where $\sigma$ runs over the permutation of $\{1, \ldots, 2 m\}$. We deduce the invariants subspace of $E$ and then that the functions (7) generate $\mathbb{C}\left[W^{\oplus n}\right]^{G}$.

Denote by Mult ${ }_{n}$ the space of multilinear maps from $V^{\times n}$ to $\mathbb{C}$ and by $\operatorname{Sym}_{n}$ the subspace of symmetric maps. For any non-negative integer $k \leqslant n$ with the same parity as $n$, let Mult ${ }_{n, k}$ be the subspace of Mult ${ }_{n}$ generated by the maps

$$
P\left(x_{\sigma(1)}, \ldots, x_{\sigma(k)}\right) \omega\left(x_{\sigma(k+1)}, x_{\sigma(k+2)}\right) \ldots \omega\left(x_{\sigma(n-1)}, x_{\sigma(n)}\right)
$$

where $\sigma$ ranges over the permutations of $\{1, \ldots, n\}$ and $P$ over $\operatorname{Sym}_{k}$. Recall that the spaces $\operatorname{Sym}_{n}$ are the irreducible representations of $G$.

Lemma 6.2. The decomposition into isotypical subspaces of Mult $_{n}$ is

$$
\text { Mult }_{n}=\bigoplus_{\substack{0 \leqslant k \leqslant n, j \\ k=n} \bmod 2} \operatorname{Mult}_{n, k} .
$$

Proof. Mult ${ }_{n, k}$ is clearly a subspace of the $k$-th isotypical component of Mult ${ }_{n}$. Since Mult $_{n}$ is the direct sum of its isotypical components, we only have to show that the spaces Mult $_{n, k}$ generates Mult ${ }_{n}$. This may be proved by induction over $n$ by using that any multilinear map symmetric with respect to the $(n-1)$ first arguments is of the form

$$
L\left(x_{1}, \ldots, x_{n}\right)=M\left(x_{1}, \ldots, x_{n}\right)+\sum_{i=1}^{n} \omega\left(x_{i}, x_{n}\right) N\left(x_{1}, \ldots, \widehat{x}_{i}, \ldots x_{n-1}\right)
$$

with $M \in \operatorname{Sym}_{n}$ and $N \in \operatorname{Sym}_{n-2}$. To show this last fact, observe that the map sending $(M, N)$ to $L$ is an isomorphism from $\operatorname{Sym}_{n} \oplus \operatorname{Sym}_{n-2}$ onto the subspace of Mult ${ }_{n}$ consisting of maps symmetric in the $(n-1)$ first arguments. Indeed, this morphism is injective by $G$-equivariance and we conclude by counting dimensions. 


\section{References}

[And] J. E. Andersen, Asymptotic faithfullness of the quantum SU( $n)$ representations of the mapping class groups. Ann. of Math. 163 (2006), no. 2, 347-368. Zbl 1157.53049 MR 2195137

[AB] M. F. Atiyah and R. Bott, The Yang-Mills equations over Riemann surfaces. Philos. Trans. Roy. Soc. London Ser. A 308 (1983), no. 1505, 523-615. Zbl 0509.14014 MR 0702806

[BHMV] C. Blanchet, N. Habegger, G. Masbaum and P. Vogel, Topological quantum field theories derived from the Kauffman bracket. Topology 34 (1995), 883-927. Zbl 0887.57009 MR 1362791

[Bul] D. Bullock, Rings of $\mathrm{SL}_{2}(\mathbf{C})$-characters and the Kauffman bracket skein module. Comment. Math. Helv. 72 (1997), no. 4, 521-542. Zbl 0907.57010 MR 1600138

[BFK] D. Bullock, C. Frohman and J. Kania-Bartoszynska, Understanding the Kauffman bracket skein module. J. Knot Theory Ramifications 8 (1999), no. 3, 265-277. Zbl 0932.57015 MR 1691437

[FLP] A. Fathi, F. Laudenbach, and V. Poénaru (eds.), Travaux de Thurston sur les surfaces. Astérisque 66-67, Soc. Math. France, Paris 1979. Zbl 0731.57001 MR 0568308

[FWW] M. H. Freedman, K. Walker and Z. Wang, Quantum SU(2) asymptotically detects mapping class group modulo center. Geom. Topol. 6(2002), 523-539.Zbl 1037.57024 MR 1943758

[Gol] W. M. Goldman, The symplectic nature of fundamental groups of surfaces. Adv. in Math. 54 (1984), no. 2, 200-225. Zbl 0574.32032 MR 0762512

[Gol] W. M. Goldman, Invariant functions on Lie groups and hamiltonian flows of surface group representations. Invent. Math. 85 (1986), no. 2, 263-302. Zbl 0619.58021 MR 0846929

[GM] F. González-Acuña and J. Montesinos-Amilibia, On the character variety of group representations in $\mathrm{SL}_{2}(\mathbb{C})$ and $\mathrm{PSL}_{2}(\mathbb{C})$. Math. Z. 214 (1993), 627-652. Zbl 0799.20040 MR 1248117

[JW1] L. C. Jeffrey and J. Weitsman, Bohr-Sommerfeld orbits in the moduli space of flat connections and the Verlinde dimension formula. Comm. Math. Physics 150 (1992), no. 3, 593-630. Zbl 0787.53068 MR 1204322

[JW2] L. C. Jeffrey and J. Weitsman, Toric structures on the moduli space of flat connections on a Riemann surface: volumes and the moment map. Adv. Math. 106 (1994), no. 2, 151-168. Zbl 0836.58004 MR 1279216

[MN] J. Marche and M. Narimannejad, Some asymptotics of topological quantum field theory via skein theory. Duke Math. J. 141 (2008), no. 3, 573-587. Zbl 1139.57030 MR 2387432

[PS] J. H. Przytycki and A. S. Sikora, On skein algebras and $\mathrm{Sl}_{2}(\mathbb{C})$-character varieties. Topology 39 (2000), no. 1, 115-148. Zbl 0958.57011 MR 1710996

[Si] A. Sikora, Character varieties. Trans. Amer. Soc., to appear; arXiv:0902.2589v3 [math.RT].

[T] V. G. Turaev, Skein quantization of Poisson algebras of loops on surfaces. Ann. Sci. École Norm. Sup. 24 (1994), no. 6, 635-704. Zbl 0758.57011 MR 1142906 
Received July 9, 2009

Laurent Charles, Institut de Mathématiques de Jussieu (UMR 7586), Université Pierre et Marie Curie - Paris 6, Paris, 75005 France

E-mail: charles@math.jussieu.fr

Julien Marché Centre de Mathématiques Laurent Schwartz, Ecole Polytechnique, route de Saclay, 91128 Palaiseau, France

E-mail: marche@math.polytechnique.fr 